\title{
IMPULSOS Y RAZONES EN EL DERECHO PENAL. HACIA UNA TEORÍA DUALISTA DE LAS EMOCIONES *
}

\author{
María Laura Manrique*** \\ CONICET, Argentina \\ laura.manrique@conicet.gov.ar
}

RESUMEN. En este trabajo defiendo una teoría dual de las emociones. Mi objetivo es mostrar que las teorías mecanicistas y cognitivas (o cognitivas evaluativas) no son rivales. Utilizo el esquema de VON WRIGHT para la relación entre mente y materia para sostener que estas teorías resuelven diferentes niveles o ámbitos de prioridades (epistémico, causal y semántico). Finalmente, subrayo que hay dos diferentes criterios para medir la intensidad de una emoción y ello puede utilizarse para explicar por qué en ocasiones las conductas emocionales agravan la pena y en otras las disminuyen.

Palabras claves: emociones, acción, derecho penal.

\section{Impulses and Reasons in Criminal Law. Through a dualistic theory of emotions}

ABSTRACT. In this paper I defend a dual theory of emotions. My main purpose is to show that mechanistic and cognitivist (or cognotivistic-evaluative) theories are not in opposition. VON WRIGHT's conception of the relationship between mind and matter is used to claim that these theories solve different areas of priorities (epistemic, causal and semantic). Finally, I underline that there are two different criteria to measure the intensity of an emotion. This distinction could be used to explain the reason why emotions sometimes render punishment more severe and others less.

Keywords: emotions, action, criminal law.

* Fecha de recepción: 18 de septiembre de 2015. Fecha de aceptación: 9 de diciembre de 2015.

** Agradezco las sugerencias y comentarios de dos evaluadores anónimos. También agradezco las sugerencias de los asistentes al seminario de Argumentación Jurídica del Instituto de Investigaciones Filosóficas (UNAM) donde discutí una versión anterior de este trabajo. En particular agradezco a Juan Antonio CRUZ Parcero, Pau Luque, Amalia Amaya, Francisca Pou, María Paula Zafón, Juan Bertomeu, Alberto Puppo, Raimundo Gama y Pablo NAVARRo sus útiles sugerencias. Por último, agradezco a Belén GulLi los cambios y sugerencias editoriales. 


\section{INTRODUCCIÓN}

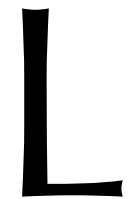

as emociones juegan un papel decisivo en la vida de los individuos. Ellas se reflejan una y otra vez en nuestros actos, puntos de vista y carácter; al igual que las creencias y las intenciones, las emociones tiñen de perspectiva y subjetividad al modo en que los agentes juegan en la compleja trama de la interacción social. Por ejemplo, en una conocida tragedia de Shakespeare, uno de sus protagonistas principales, Yago, manipula una tensa situación social; su conducta está determinada por la ira y el resentimiento que siente hacia sus colegas (Casio, Rodrigo, etc.). De ese modo, él urde una trama en la que los celos conducen a Otelo a estrangular a Desdémona. En este contexto, las emociones juegan un papel central y comprendemos acabadamente esa situación caracterizada por Shakespeare porque esas emociones en las que se basa el relato son familiares; son rasgos usuales que, con frecuencia, acompañan nuestro comportamiento. Por ello, también somos capaces de evaluar la conducta de Yago y de Otelo (e.g., sentir cólera o compasión), como una suerte de proyección de nuestras propias expectativas de conducta en situaciones similares ${ }^{1}$.

A pesar de su importancia y a diferencia de las intenciones y las creencias, las emociones han sido sistemáticamente descuidadas en la teoría jurídica. Probablemente, esta desatención tenga que ver con el hecho de que las emociones son el objeto propio de otras disciplinas específicas (e.g., psicología) y de allí podría surgir la impresión de que no corresponde al jurista lidiar con esos problemas. Pero, aunque fuese verdad que las investigaciones empíricas sobre las emociones son el patrimonio específico de otras disciplinas, ello no debería impedir avanzar sobre importantes cuestiones conceptuales y normativas que subyacen a la conexión entre emociones y la atribución de responsabilidad moral y jurídica. Después de todo, si las emociones ocupan un lugar central en nuestras vidas, también tendrán incidencia en el modo en que se cometen los delitos y en su consecuente responsabilidad.

Las emociones poseen diferentes ámbitos de impacto en el derecho. Por ejemplo, podría considerarse el papel que las reacciones emocionales de las víctimas y ciudadanos juegan al momento de castigar ciertos crímenes, así como también el modo en que las emociones determinan a un agente en la ejecución de una conducta disvaliosa. Ambas perspectivas, que podrían ser respectivamente denominadas como «respuestas emocionales» $\mathrm{y}$ «acciones emocionales», son de indudable relevancia, pero - como señala Victor TADROSS (2004: 67, 332)

no parece que pueda lograrse demasiado progreso en la determinación del alcance de la responsabilidad penal mediante la consideración de la emociones en el contexto del castigo. Eso sería poner la carreta delante de los caballos.

Por ello, en este trabajo no abordaré el primer grupo de cuestiones (e.g., las respuestas emocionales) y me limitaré a explorar la conexión entre acciones emocionales y la responsabilidad penal. Sin embargo, dar lugar a las emociones en la responsabilidad penal genera un problema ya que, por una parte, en algunas ocasiones las acciones emocionales son consideradas como un acontecimiento incontrolable para el agente

${ }^{1}$ Sobre la conexión entre Otelo y emociones vid., por ejemplo, ELSTER, 1998: 109; SCHIMILL, 2010. 
mientras que, por otra parte, en ciertas circunstancias las emociones agravan el modo en que se ejecuta una conducta disvaliosa. Esta doble faceta de las emociones sugiere que ellas son: i) impulsos que el agente no puede resistir, que mecánicamente conducen a un resultado penalmente típico, pero también ii) que las emociones integran el conjunto de razones que determinan la conducta del agente y que, por consiguiente, el agente puede razonablemente controlar.

Mientras que los enfoques clásicos de las emociones (genéricamente denominados teorías mecanicistas) subrayan la tensión entre razón y pasión, las concepciones contemporáneas (genéricamente denominadas teorías cognitivo-evaluativas) arrojan luz sobre el modo en que las emociones pueden ser racionalizadas y formar parte de nuestras razones para la acción. En gran medida, el debate filosófico sobre las emociones gira en torno a los argumentos para establecer una prioridad de una concepción sobre su rival. En este sentido, la controversia gira en una suerte de disputa a «todo o nada». Sin embargo, como intentaré mostrar en este trabajo, esta presentación del debate se construye sobre una falsa antinomia. Más bien, es necesario discriminar entre diversas relaciones de prioridad (i.e.e, epistémica, causal y semántica) y ello sirve para disipar la imagen de una oposición completa y radical entre teorías mecanicistas y cognitivoevaluativas. También, trataré de mostrar algunas consecuencias específicas para el derecho penal que se derivan del reconocimiento de una teoría dual de las emociones. En particular, sostendré que en muchos diseños institucionales, donde las emociones suelen ocupar un lugar tanto para agravar como para atenuar la pena, no es que se utilicen dos teorías acerca del concepto de emoción sino que existen dos sentidos en los que hablar de intensidad de una emoción.

\section{TEORÍAS MECANICISTAS Y TEORÍAS COGNITIVO-EVALUATIVAS}

Un análisis sistemático de las emociones es todavía una asignatura pendiente en la filosofía analítica contemporánea. Así, en palabras de LyONs (1993: 1-2),

probablemente sea cierto que en la actualidad la emoción es un tema relativamente relegado por los filósofos. Cuando se estudia, lo corriente es atender a problemas específicos a expensas de cualquier intento encaminado a mostrar cómo dichos problemas podrían estar interrelacionados o cómo los enfoques de dichos problemas podrían afectar nuestra concepción global de la naturaleza de la emoción.

En cierta medida, esta fragmentación es una consecuencia de la heterogeneidad del objeto de estudio, que abarca tanto a ciertas emociones «básicas» (e. g., miedo, ira, odio, compasión, repugnancia, etc.) como también a otras de naturaleza «complejas» (i.e. aquellas que ejemplifican una combinación de emociones básicas). De igual modo, es usual distinguir entre «emociones primarias» que podrían ubicarse en un estadio evolutivo más primitivo (e.g., comunes a todos los miembros de la especie) y las «emociones secundarias» que están asociadas a un desarrollo social o cultural (e. g., admiración ante bienes culturales) (GONZÁLEZ LAGIER, 2009: 75-76). Sin dudas, esta clasificación es demasiado rudimentaria para dividir de manera exhaustiva y excluyente a todo el campo emocional. Sin embargo, en la medida en que encontremos ejemplos paradigmáticos de ellas, puede servir como guía para el análisis. En lo sucesivo, me centraré en emociones básicas y dejaré de lado, salvo mención en contrario, otras 
emociones que requieren de adiestramiento, desarrollo social, o logros educativos y culturales.

\subsection{Teorías mecanicistas}

A pesar de la carencia de enfoques sistemáticos, es posible encontrar importantes ideas defendidas a lo largo de la historia por los filósofos clásicos y modernos más importantes (ARistóteles, Descartes, Hobbes, Hume, Spinoza, etc.). Por ejemplo, una de la más influyente caracterización de las emociones se remonta hasta los diálogos de PLATÓN y subraya que ellas son básicamente fuerzas o impulsos que experimentan los agentes y que los guían (de manera irracional) a la acción. Su famosa metáfora del auriga (la razón) conduciendo un carro arrastrado por dos caballos (motivaciones y emociones) señala claramente una línea de análisis que ahora resulta clásica: la oposición entre razón y emociones ${ }^{2}$. Esta es una de las ideas características de las Teorías Mecanicistas: las emociones nos suceden; son cosas que nos ocurren, es decir, están fuera de nuestro control y por ello juegan en contra de una conducta racional (GonZÁlez LAGIER, 2009: 49). Probablemente, ninguno de los exponentes clásicos y contemporáneos de esta concepción suscriba sin reservas un grupo común de notas definitorias ${ }^{3}$. En este sentido, sus propuestas reflejan solo un cierto aire de familia, que destacaré brevemente a continuación.

En general, las concepciones mecanicistas identifican a las emociones a partir de eventos fácticos (fisiológicos, psicológicos, etc.) específicos. Por ejemplo, para DeSCARTES, las emociones están intrínsecamente ligadas a sensaciones de los agentes, o como ocurre en la propuesta de JAMES-LANGE, los cambios fisiológicos (corporales) son el criterio para identificar a una emoción ${ }^{4}$. Sin esos cambios la emoción sería simplemente algo vacío, un episodio meramente intelectual. En el ámbito del derecho penal es usual que jueces, abogados y peritos recurran a criterios similares para acreditar una determinada emoción. Así, por ejemplo, al analizar los supuestos de «emoción violenta» se utilizan expresiones tales como «alteración mnemónica», «estado crepuscular psíquico», «islotes amnésico», etc. ${ }^{5}$. En todos estos casos, esos cambios ocurren más allá del control racional del agente; en un cierto sentido él no sabe lo que hace (y de allí que sea usual también señalar que no recuerda - o no recuerda claramente- lo que hizo).

Especial interés en el amplio campo de las versiones mecanicistas merecen la versión psicologista defendida por David Hume en su Tratado de la naturaleza bumana. Allí, en el segundo libro, HuME recoge las conclusiones generales elaboradas en el pri-

2 Vid. Platón, «Fedro», en P. DE AzCÁRATe (ed.), Platón. Obras Completas, 1871: II, Madrid: Medina y Navarro.

3 De hecho, no es infrecuente encontrar en los filósofos clásicos (e. g. ARISTÓtelEs, HumE, etc.) argumentos para clasificarlos tanto en las concepciones mecanicistas como en sus alternativas rivales.

${ }_{4}$ Aproximadamente en la misma época que Williams JAMES presentaba su concepción cartesiana de las emociones, un autor de origen danés, C. G. LANGE, trabajaba en la misma idea. Por esa razón, esta versión que menciono suele denominarse JAMES-LANGE. Vid. R. SOLOMON, 2003: 65.

5 La emoción violenta es uno de los modos establecidos en el art. 81, inciso a), del Código Penal argentino para disminuir la responsabilidad en caso de homicidio, pero la fórmula del código es más compleja ya que exige expresamente «emoción violente que las circunstancias hiciesen excusables». 
mer volumen del tratado acerca del origen y división de las ideas. Para él, las emociones son impresiones en el alma dadas por ciertas asociaciones con sensaciones habituales y, ciertas ideas correlativas. Así, la emoción es la sensación en el alma y las restantes cosas son integrantes de una cadena causal que se deriva de la emoción (LYONS, 2009: 18).

Así, el orgullo es una sensación placentera, y la humildad lo es dolorosa; y si eliminamos el placer o el dolor, no queda en realidad ni orgullo ni humildad. Nuestro mismo sentimiento es quien nos convence de esto, y más allá de nuestro sentimiento es vano razonar o disputar (Hume, 1739: II, Parte Primera, Sección V).

A diferencia de las concepciones de Descartes o James-Lange, Hume sostiene que lo que distingue unas emociones de otras son las impresiones en el alma y no los cambios fisiológicos. Para este autor únicamente emociones violentas como el odio o el amor están correlacionados con cambios físicos. Sin embargo, con esta concepción no parece fácil caracterizar adecuadamente a ciertas emociones que no se asocian inmediatamente con el placer y el dolor (e.g., la esperanza, el hastío, etc.). Pero, a pesar de ello, es importante señalar que HumE es uno de los primeros autores en: i) destacar que las emociones poseen un objeto e insistir en cuáles son las ideas que las causan, y ii) tratar de analizar detalladamente el vínculo entre emociones y acciones ${ }^{6}$.

La conexión entre emociones y acciones ha sido uno de los temas centrales de las diferentes versiones conductistas (e. g., WATSON, SKINNER). A su vez, fue Gilbert RYLE quien, a partir de un capítulo específico de su célebre texto sobre el concepto de lo mental, tuvo el mérito de reavivar en el siglo XX, el interés por las emociones en el ámbito de la filosofía analítica ${ }^{7}$. Su propósito era descartar una concepción tradicional que reservaba para las emociones un ámbito interno, privado e inaccesible y que, de alguna manera, causaba las acciones. En su opinión, por el contrario, las emociones tienen que explicarse a la luz de las disposiciones de conducta y, dado que las disposiciones no son eventos, la explicación de la conexión causal entre emociones y acciones tenía que ser reformulada (RYLE, 1949: 86-87).

Aunque todas estas concepciones mecanicistas capturan intuiciones importantes, ellas no explican adecuadamente las conexiones entre emociones, sensaciones, percepciones, motivaciones y acciones. Por ejemplo, si las emociones fuesen -como señalaba DESCARTES - sensaciones y sentimientos subjetivos (i.e. impresiones que ciertos objetos dejan en nuestro cuerpo), entonces parece que no habría lugar para equivocarse acerca de las emociones que tenemos. Si sentir miedo es tener miedo, entonces siempre tendré conciencia de la emoción que experimento. A su vez, si aceptamos - como propone la concepción de JAMES-LANGE - que la percepción de una reacción fisiológica específica define la emoción, entonces no habría lugar para que dos emociones diferentes puedan generarse en las mismas reacciones fisiológicas ${ }^{8}$. Una crítica similar puede elaborarse contra las versiones conductistas de WATSON y

6 Al respecto, vid. A. Kenny, 2003: 66-69; D. GonzÁlez Lagier, 2009: 31. Aun cuando Hume ha señalado expresamente que el volumen sobre la moralidad es relativamente independiente de los dos primeros volúmenes, existe un consenso dominante acerca de sus importantes conexiones. Para una discusión general acerca de la conexión que HuME establece entre las emociones, la acción y la moral, vid. S. SUTHERLAND, 1976: 26: 14-23, y la bibliografía allí citada.

7 Vid. RYle, 1949: 83-115. También: Ryle, 2009: 284-299.

8 En el mismo sentido: LyONS, 2009: 13-16; SOLOMON, 2003: 67; GONZÁLEZ LAGIER, 2009: 35-36. 
SKINNER ya que ellos no podrían explicar que ante la misma emoción dos sujetos pueden reaccionar de diferente manera. Por ejemplo, en un caso de profundo disgusto o enojo, un agente puede gritar airadamente y otro, por el contrario, solo ensimismarse (GONZÁlez LAGIER, 2009: 41). Finalmente, la concepción de RYLE, podría cuestionarse porque no deja aparentemente espacio a la distinción entre tener una emoción y fingir una emoción, o por eliminar la referencia a estados mentales al momento de dar cuenta de las emociones.

Por supuesto, hay varias estrategias para dar cuenta de estas objeciones y no es posible discutir aquí acerca de sus méritos o defectos. Más bien, me interesa establecer una suerte de geografía conceptual de la discusión, con el propósito de dejar claro que, como señalaba HART (1963: 1-2) acerca de las discusiones conceptuales en teoría del derecho, estas disputas filosóficas muchas veces surgen como consecuencias de resaltar exageradamente distintas intuiciones importantes. Estas intuiciones podrían resumirse en las siguientes ideas:

En primer lugar, las propuestas mecanicistas recogen la conexión entre emociones y pasividad, i. e., las emociones se experimentan como algo que nos invade sin que seamos capaces de consentir o controlar. Segundo, y derivada de la idea anterior, esta propuesta captura la intuición de que las emociones son algo externo a la persona. Finalmente, esta propuesta recoge la idea de que las emociones son algo con un grado variable de intensidad; y, en ciertas situaciones, se piensa en ellas como fuerzas imposibles de repeler ${ }^{9}$.

\subsection{Teorías cognitivo-evaluativas}

Esta familia de teorías ejemplifica la concepción dominante en la actualidad. En general, ellas sostienen que las emociones están asociadas a nuestras valoraciones ya que solo frente a objetos de cierta importancia reaccionamos emocionalmente. Por ejemplo, no nos enojamos ante cosas que nos parecen irrelevantes o no sentimos miedo si creemos que la amenaza no es suficientemente seria. Así, las emociones se distinguen a partir de creencias o valoraciones de ciertos objetos por parte de un agente. En general, uno de los rasgos más destacados de estas propuestas es que, a diferencia de las teorías mecanicistas, ellas admiten la racionalidad de las emociones. Ello significa que las emociones desempeñan un papel central en el razonamiento práctico de un agente $\mathrm{y}$, en ciertas circunstancias, determinan intencionalmente su comportamiento.

Es importante remarcar, aunque parezca obvio, que las acciones emocionales son, en primer lugar, acciones; es decir pautas de conductas ejecutadas por un agente. La explicación de estos eventos (ya sea que se favorezca un esquema causal o un esquema intencional) requiere identificar un objeto de intencionalidad, que es definido a partir de un complejo epistémico-volitivo. Las emociones conforman ese complejo ya que surgen a partir de evaluaciones que el agente realiza y ellas acompañan a sus motivaciones y creencias acerca de lo que exige una situación. Conforme a esta idea, las

9 Kahan y Nussbaum, 1996: 280. Retomaré la cuestión de la intensidad de la emoción en el último apartado. 
emociones también pueden analizarse desde un punto de vista externo y, en este caso, el observador puede calificar a una emoción como razonable, correcta, etc. En este caso, el agente ha sido «movido a la acción» por un conjunto aceptable de elementos determinantes de su conducta.

No es posible presentar aquí cada una de las variantes de esta concepción y, a efectos de ilustrar de manera esquemática los contornos de una propuesta específica, expondré a continuación los rasgos básicos de una de las propuestas más conocidas: la concepción de William LyONS, quien defiende una teoría cognitiva (que denomina causal-evaluativa). Para este autor:

$\mathrm{X}$ se considera un estado emocional si y solo si $\mathrm{X}$ es un estado fisiológicamente anormal, causado por el sujeto que, en tal estado evalúa su situación [...]. La emoción es un estado psicosomático, un estado corporal causado por una actitud, en nuestro caso una actitud evaluativa (LYONS, 2009: 79).

Su teoría está comprometida con los siguientes enunciados:

a) Los estados emocionales son considerados elementos fácticos y no como disposiciones o propensiones a poseer determinado estado.

b) Este estado emocional conlleva una evaluación y esa evaluación causa ciertos cambios fisiológicos (anormales) en el sujeto.

c) Las emociones se diferencian unas de otra por el aspecto evaluativo.

d) El concepto de estado emocional puede abarcar deseos, evaluaciones o cambios fisiológicos.

e) El aspecto evaluativo provoca la conducta emocional por una conexión causal y racional con los deseos. Ello significa que la evaluación más el deseo forman una razón para actuar y ella, a su vez, causa la acción (o conducta emocional) del agente.

f) Aunque el aspecto evaluativo sea el central ello no significa que las emociones sean algo intangible y no objetivas (LyONs, 2009: 73-94).

Entre las críticas usuales a estas propuestas es posible destacar a las siguientes. En primer lugar, parecen comprometerse con una perspectiva reduccionistas ya que las emociones son reducidas a combinaciones de creencias y deseos; identificándolas así con los estados intencionales paradigmáticos. En segundo lugar, en ocasiones estas reconstrucciones no prestan suficiente atención al aspecto fenomenológico y ofrecen, por tanto, una imagen excesivamente racional de las emociones. Por último, estas propuestas no distinguen adecuadamente entre la satisfacción emocional y la satisfacción intencional ya que el objeto intencionalmente perseguido está uniformemente configurado. Pero, parece claro que en ciertas ocasiones, deseamos conseguir ciertas cosas (e. g., infligir un mal a un enemigo) movidos por emociones específicas (e.g., animo de venganza) y no hay que descartar que una vez conseguida la satisfacción intencional (infligir el mal), el agente se sienta emocionalmente insatisfecho (no ha conseguido aplacar su sed de venganza) ${ }^{10}$.

A pesar de estas dificultades, esta familia de teorías posee un innegable atractivo para cuestiones vinculadas con la responsabilidad penal ya que ellas introducen la posibilidad de evaluar como razonable o irrazonable una emoción y su reacción. Dos

${ }^{10}$ Un análisis de estos problemas se encuentra en GONZÁLEZ LAGIER, 2009: 49-51. 
autores que han prestado especial atención a esta relación son Martha NuSSBAUM y Dan KAHAN (KAHAN y NusSBAum, 1996: 269-374). Ellos sostienen, que así como las emociones son resbaladizas en la vida también lo son en el derecho penal y, mediante la revisión de numerosos casos de derecho penal americano, muestran que la jurisprudencia ha oscilado continuamente entre una teoría cognitivo-evaluativa y una teoría mecanicista. Sin embargo, en este artículo, ellos defienden que una teoría cognitivoevaluativa no solo es descriptivamente más adecuada para dar cuenta del modo en que funcionan las emociones sino que también sostienen que el mejor modo de incorporar las complejidades de las emociones en el derecho penal es a través de una teoría cognitiva.

En general, ellos sostienen que una teoría cognitivo-evaluativa es superior porque es más eficiente en prevenir y condenar delitos y, sobre todo, porque es mucho más honesta que la teoría mecanicista que pretende disfrazar las discusiones morales (KAHAN y NuSSBAUM, 1996: 274). También sostienen, al igual que todas las teorías cognitivas, que ciertas actitudes cognitivas son condición necesaria para generar una emoción; las creencias son una parte constitutiva de la emoción y no son causas externas. La razón es que para distinguir una emoción de otra no se puede evitar mencionar la creencia que se encuentra involucrada. Finalmente, señalan que ciertas creencias relevantes son suficientes para generar una emoción. Todos los ejemplos que se nos ocurran de personas que posean una creencia y no generen la emoción (por ejemplo, dolor por la muerte de un hijo) es porque en el fondo no poseían la creencia (i. e., se niegan a aceptar la perdida) (KaHAn y Nussbaum, 1996: 294) ${ }^{11}$.

A pesar que la propuesta de NUSSBAUM y KAHAN ha tenido una amplia repercusión, no es obvio que su propuesta pueda superar las críticas que se dirigen a este grupo de concepciones en general. Por ejemplo, no es fácil resolver con una teoría como la de NuSSBAUM y KAHAN los casos en los que un agente no siente la emoción específica en situaciones en las que es razonable desarrollar esa respuesta emocional. Así, frente a una agresión sería razonable sentir temor y, luego, invocar como una excusa el hecho de haber actuado impulsado por el miedo. Sin embargo, la teoría exagera el papel de las creencias y las evaluaciones, disminuyendo la relevancia de las sensaciones. Puede ocurrir que un agente no sienta miedo ante la agresión. En ese caso, una teoría mecanicista mantendría la responsabilidad del agente, pero la teoría cognitivo-evaluativa tendría que admitir que su conducta estaba excusada.

Es momento de detener la exploración de las diferentes propuestas conceptuales acerca de las emociones. El objetivo de este breve recorrido no tenía por función repetir cosas que otros autores han expuesto con mayor detalle sino de exhibir la agenda de problemas más importantes que aún aguardan solución. Tres moralejas pueden extraerse de este ensayo de una geografía de las concepciones de las emociones. En primer lugar, es evidente que las emociones ocupan un lugar cada vez más relevante en las discusiones sobre temas centrales para la atribución de responsabilidad penal (e. g., teoría de la acción, justificación del castigo, etc.). En segundo lugar, la oposición entre las dos grandes corrientes filosóficas acerca de las emociones es básicamente

11 Años más tarde NusSBAum volvió a dedicarse a cuestiones vinculadas a las emociones y el derecho. Sin embargo, sus ideas no parecen haberse modificado sustancialmente. Vid. NussBaum, 2006: 41. 
una disputa acerca de la racionalidad de las emociones y su eventual potencial para intervenir en el razonamiento práctico de los agentes. Finalmente, existe un profundo desacuerdo sobre las ventajas y desventajas de cada propuesta y ello repercute sobre diferentes puntos de vista acerca de sus respectivas primacías conceptuales. En lo que resta de este trabajo trataré de explorar una manera de disolver esta polémica. En particular, sostendré que no hay un único tipo de prioridad que se pueda establecer entre ellas y que, por esa razón, no tiene sentido una polémica, en términos absolutos, acerca de qué teoría es mejor.

\section{LA CONCEPCIÓN DUALISTA DE LAS EMOCIONES}

En esta sección, en base a ciertas ideas de VON WRIGHT sobre la conexión entre mente y materia defenderé una concepción dualista de las emociones ${ }^{12}$. En mi opinión, esta concepción dualista explica de manera más adecuada las relaciones entre aspectos internos y externos de las emociones (VON WRIGHT, 1998: 147 y 58-60). Al igual que las acciones no pueden identificarse únicamente con fenómenos mentales o conductuales, las emociones son un fenómeno complejo y ellas no pueden reducirse ni a eventos sensoriales o fisiológicos (como hace la teoría mecanicista) ni a estados mentales (como parecen favorecer las teorías evaluativas). Por supuesto, no pretendo sostener que las emociones sean similares a las acciones o conductas en todos sus aspectos. Más bien, intento destacar que emociones y acciones comparten un rasgo en común que vale la pena resaltar: los aspectos internos (e.g., mentales o volitivos) y externos (e.g., cambios en el mundo).

La polémica acerca de la naturaleza de la acción ocupa un lugar central en la filosofía de la acción contemporánea. Uno de los aspectos más debatidos es acerca de la validez del esquema de explicación de acciones y dos concepciones (causal y teleológica) compiten recurrentemente en este ámbito ${ }^{13}$. Los modelos de explicación teleológica subrayan que la relación entre acción y resultado es de naturaleza conceptual y, por ello, al no existir independencia lógica entre ambos eventos, no es posible establecer una relación de causalidad. Uno de los representantes más destacados de esta línea de análisis es Georg Henrik VON WRIGHT. Para este autor, una acción involucra una transformación de estado de cosas, por ejemplo, por medio del comportamiento del agente el mundo cambia desde un cierto estado (e. g., en el que una puerta está cerrada) a otro estado diferente (e.g., en el que esa puerta está abierta). Este estado final del cambio es el que nos permite identificar una cierta acción. Así, la acción de abrir la puerta se define por el estado de cosas de que la puerta resulte abierta. Como consecuencia de ese estado de cosas pueden producirse otros eventos (e. g., el descenso de la temperatura en una habitación) y, en ese caso, la conexión entre el resultado de una acción y sus consecuencias es de naturaleza causal (VON WRIGHT, 1970: 56).

12 Con cierta cautela, este enfoque podría denominarse «cartesiano» ya que se asienta en un dualismo irreductible entre aspectos mentales y conductuales. Sin embargo, dado que DESCARTES es considerado como un partidario de las concepciones mecanicistas, parece mejor utilizar la expresión «concepción dualista».

13 Dos de los teóricos más representativos de esta disputa son Donald DAVIDSON y Georg Henrik vON WRIGHT. Para una discusión sobre la relación entre acción y resultado vid., por ejemplo, DAVIDSON, 1980, y VON WRIGHT, 1980. 
Sin embargo, si se admite que la relación entre resultados y consecuencias es causal, entonces parece intuitivo asumir que esas relaciones causales también juegan un papel determinante en el modo en que se produce el resultado. En otras palabras, es tentador suponer que las redes causales son inescapables y que los aspectos internos que determinan la acción son también parte de un proceso de eventos y estados de cosas causalmente aptos para explicar cambios en el mundo. Una de las maneras de desarrollar este argumento es reducir los estados mentales a fenómenos neuronales, que se conectan causalmente con nuestros movimientos corporales y los cambios externamente perceptibles. Así, aunque los estados internos no serían directamente observables, ello no implicaría que tengan una naturaleza diferente a otros fenómenos empíricos (VON WRIGHT, 1998: 126).

Sin embargo, VON WRIGHT rechaza este argumento reduccionista y asume la «carga cartesiana» del dualismo. Está fuera de discusión que las explicaciones causales son apropiadas para dar cuenta de: i) la conexión entre resultados y consecuencias, y ii) la conexión entre eventos neuronales y movimientos corporales. Pero, ninguna de estas concesiones es relevante para dirimir si los estados mentales son fenómenos fácticos. $\mathrm{Al}$ referirse a las acciones, él sostiene que la conexión entre el aspecto interno y externo de la acción es más compleja de lo que normalmente asumimos. Por una parte, aunque los cambios neuronales no sean directamente observables en la mayoría de los casos, nada impide suponer que un investigador puede obtener datos en «tiempo real» de lo que ocurre en nuestros cerebros cuando ejecutamos una cierta acción. En este sentido, este aspecto neuronal de la conducta sería únicamente una parte más elaborada del aspecto externo del comportamiento de un agente. No hay en este presupuesto nada lógicamente inadmisible o extraño. Por otra parte, esos cambios neuronales son «lógicamente secundarios» frente a los estados mentales con los que ellos están correlacionados. Podría decirse que esos estados mentales supervienen a los cambios físicos (fisiológicos, neuronales, etc. ${ }^{14}$.

Por supuesto, gran parte de la filosofía de la acción se ha dedicado a establecer el modo en que se producen estas conexiones entre eventos y correlaciones entre estados mentales y sucesos. Así, algunos reducían la conexión de la mente a la materia (como HOBBES) y otros idealistas de la materia a la mente (BERKELEY). Sin embargo, para VON WRIGHT, esta conexión es entendida de mejor manera como algo dual, como un fenómeno en el que los estados mentales interactúan con las funciones que cumple el sistema nervioso de un agente y se plasman en una conducta externa que interpretamos como una acción específica (VON WRIGHT, 2003: 43-44). Así, formarse una intención de actuar genera cambios neuronales que a su vez producen determinado comportamiento y, del mismo modo, un estimulo externo produce cierto cambio neuronal que a su vez genera determinada sensación (VON WRIGHT, 2003: 45). Con respecto al modo en que se produce este proceso VON WRIGHT (2003: 46) sostiene:

La naturaleza «misteriosa» de la transición del proceso neuronal a la sensación mental es responsable, creo yo, de la tentación de decir que la sensación y la manifestación neuronal que está allí cuando el sujeto tiene la sensación, son en algún sentido, lo mismo o idénticas. Y hay una tentación similar en identificar el trasfondo mental de la voluntad de

14 Sin lugar a dudas, este relato acerca de los procesos neuronales y fisiológicos es rudimentario, pero su admitida simplicidad, creo, no priva de su valor heurístico al análisis. VON WRIGHT, 1998: 126. 
una acción con los estados neuronales y los procesos que son causalmente responsables de la parte conductual de su aspecto externo.

En definitiva, quienes asumen la «carga cartesiana» — como VON WRIGHT-pueden afirmar que hay una indudable relación causal entre eventos neuronales y los cambios externos en el mundo, pero también pueden negar que la explicación de una acción quede completa por el solo hecho de haber establecido esos nexos causales. Una acción es, en este sentido, algo más que el comportamiento fáctico del agente.

Para evitar el error reduccionista VON WRIGHT propone un modo de establecer las interconexiones de tres aspectos centrales vinculados a la acción, e. g. el neuronal, el mental y el conductual. La conexión de estos aspectos se produce de tres diferentes relaciones conceptuales, $i$. e. epistémica, causal y semántica (VON WRIGHT, 2003: 46).

a) La prioridad epistémica. En primer lugar, lo mental es epistémicamente prioritario sobre lo neuronal. En este sentido, para poder conocer el impacto que tiene cierta emoción en el cerebro, o cualquier otro estado mental, primero debemos identificar el fenómeno de un modo independiente. Por ejemplo, no podremos identificar lo que el miedo produce en el cerebro al menos que tengamos previamente en claro cuándo un cierto individuo siente (tiene) miedo.

b) La prioridad causal. En otro nivel, existe una prioridad causal de lo neuronal sobre lo conductual. Parece más allá de discusión que primero se producen ciertos «cambios» a nivel cerebral y fisiológico antes de que la conducta sea realizada. Si fuese posible inspeccionar lo que ocurre en el sistema nervioso de un individuo, entonces veríamos que sus movimientos corporales se producen instantes después de ciertos cambios neuronales y fisiológicos. Así, el movimiento del individuo al abrir una puerta es parte de lo que ocurre luego de que su sistema nerviosos procese ciertos estímulos. ${ }^{15}$

c) La prioridad semántica. Existe una prioridad semántica de la conducta sobre lo mental. Esto quiere decir, en palabras de VON WRIGHT que

las reacciones conductuales causadas por los equivalentes neuronales de los diversos estados mentales constituyen lo que significa decir que el sujeto experimenta («sufre») esos estados (VON WRIGHT, 2003: 47).

El objetivo de VON WRIGHT está dirigido a echar luz sobre la conexión entre mente y materia. Este es un problema filosófico de gran magnitud que no trataré aquí. Me interesa ahora resaltar las similitudes de esta discusión con las emociones. Así como existen teorías reduccionistas en cuanto a la conexión entre mente y materia para la explicación de la acción, creo que tanto las tesis mecanicistas como las cognitivas evaluativas son reduccionistas para comprender las conexiones internas y externas en las emociones.

Así, una teoría mecanicista (sobre todo en sus versiones más radicales) reduce las emociones a las conexión causal que existe entre lo neuronal y lo conductual, distor-

15 Una teoría a nivel neurofisiológico de gran riqueza en este nivel es la teoría de Antonio DAMASIO. Este autor pretende mostrar no solo que las emociones juegan un papel en la toma de decisiones sino que también ellas son necesarias para que el agente tome decisiones racionales. Su teoría posee una gran riqueza y utilidad. Por esa razón no creo que sea este el lugar para dedicarle el lugar que se merece en «una teoría de las emociones». Solo deseo mencionar que su postura se ubicaría en este nivel causal pues él nos ayuda a entender cómo funciona el cerebro y que las emociones no pueden ser identificadas solo en un sector de este. Vid., por ejemplo, DAMASIO, 1994. 
sionando las otras maneras de conectar los aspectos conductual y mental. Al reducir las acciones emocionales a eventos causales, la teoría refleja un aspecto de las emociones pero deja de lado otros elementos relevantes para entender situaciones en las que las emociones funcionan como fundamento de las acciones.

Las teorías mecanicistas (en su versión conductista) insisten en la prioridad semántica que posee la conducta sobre lo mental. Estas teorías, sin embargo, olvidan la importancia de las conexiones epistémicas y causales para explicar las emociones. Al examinar determinada conducta asumimos que el sujeto padecía ciertas emociones o intentaba realizar esta conducta por ciertas razones. El problema central, si no se tienen en cuenta las otras relaciones, es que ciertos comportamientos pueden parecer que fueron realizados por cierta razón (temor) pero a pesar de ello fueron ejecutados por otra diferente (venganza). Por ejemplo, una persona de gran fortaleza me ataca y reconozco que se trata de un antiguo enemigo. Aunque tengo razones para entrar en pánico, supongamos que soy temeraria, a pesar de la seriedad de la amenaza, me mantengo tranquila. Más aún, supongamos que aprovecho una pequeña oportunidad de la lucha para deshacerme de mi enemigo. Desde una perspectiva externa, el observador del evento puede aceptar que mi conducta es ejecutada por «miedo insuperable», y, sin embargo tanto a nivel mental como neuronal ella significó algo diferente ${ }^{16}$.

Por último, podría sostenerse que las teorías cognitivas al insistir en el aspecto voluntario de las emociones ponen demasiado acento en la prioridad epistémica que existe entre lo mental sobre lo neuronal. Sin embargo, estas teorías poseen un aspecto normativo en el que enfatizan también las condiciones para que una emoción cuente como razonable o irrazonable. El aspecto voluntario de la emoción es utilizado como presupuesto. Este aspecto evaluativo podría considerarse más bien como un dato semántico (interpretativo). En otras palabras, ante un desacuerdo, entre el punto de vista del agente y lo que significa cierta conducta emocional en un determinado contexto, elegirán la segunda.

A simple vista podría criticarse estas interconexiones por ser circulares. Sin embargo ellas son de diferente naturaleza algunas relaciones son empíricas y otras de naturaleza conceptual. En síntesis, estas relaciones nos permiten ver por qué ambas teorías son necesarias para explicar la misma conducta emocional.

\section{INTENSIDAD DE LA EMOCIÓN. LA EMOCIÓN COMO AGRAVANTE Y ATENUANTE EN DERECHO PENAL}

En los párrafos anteriores intenté mostrar que las teorías tradicionales de la emoción no son rivales. Sino, por el contrario, ambas reflejan diferentes niveles o ámbitos en los que funcionan las emociones. En este apartado pretendo esbozar un argumento para mostrar que no hay nada incorrecto o irracional en los códigos penales cuando en ocasiones las emociones sirven para agravar el delito (odio racial) y en otras sirven para

${ }_{16}$ Por supuesto, existen emociones que no se reflejan en la conducta de los agentes y esto genera un problema mayor para las teorías de las emociones. Sin embargo, el objeto central del derecho penal es la evaluación de ciertas conductas, es por ello que no me ocuparé aquí de analizar las emociones como fenómenos que no se reflejan en acciones. 
atenuarlo (emoción violenta). Sin embargo, esto no quiere decir, como sostiene GoNZÁLEZ LAGIER, que en ocasiones se defienda una teoría mecanicista y, en otras, una teoría cognitiva (GONZÁLEZ LAGIER, 2009: 145 y ss.). Tampoco, como sostiene NusSBAUM y KAHAN en que el mejor modo de entenderlo es reconstruyendo los agravantes y atenuantes bajo la teoría cognitivo evaluativa (KAHAN y NuSSBAUM, 1996: 327).

En este apartado sostendré que hay dos criterios para medir la intensidad de la emoción y no el concepto de emoción misma. Y estos diferentes modos de medir la intensidad de la emoción explican la manera en que las emociones funcionan en muchos códigos penales.

Aunque a simple vista resulte desconcertante, diferentes criterios pueden utilizarse para medir un mismo concepto. Imagine que quiero clasificar a un grupo de personas en «grandes» y «pequeñas». Esta clasificación puede llevarse a cabo utilizando dos criterios, el de altura y el de la edad, algunas de las personas clasificarán como «grandes» bajo los dos criterios y otras lo harán bajo alguno o ninguno. Esto no significa que existan dos conceptos de «tamaño». Para decirlo de un modo concreto, los diferentes criterios para medir el tamaño o la intensidad de la emoción son la concepción que poseemos acerca del concepto de tamaño o de emoción y no diferentes conceptos. (DWORKIN, 1977: 212 y ss.).

Con respecto al tema que interesa aquí, esta distinción tiene que ver con: a) la intensidad de la emoción como sentimiento, y b) la intensidad de la emoción como motivo (KENNY, 2003: 24). En lo que sigue reconstruiré brevemente esta distinción e intentaré mostrar cómo funciona en el ámbito del derecho penal.

La intensidad en su primer criterio se mide teniendo en cuenta la potencia o fuerza de ciertos cambios físicos, e. g. modificaciones de ciertas expresiones faciales, gestos, posturas y reacciones. Por ejemplo, para medir la intensidad de la vergüenza que siente una persona se puede analizar, entre otros rasgos, el mayor o menor nivel de sonrojo ante cierta situación.

Un parámetro de intensidad de la emoción, en este sentido, posee incidencia en la atribución de responsabilidad y el derecho penal. Por ejemplo, para CARRARA la disminución del reproche en casos emocionales se produce por «la violencia ejercida sobre la voluntad del agente aún por una fuerza meramente interna aunque sea viciosa» (SOLER, 1956: 60). Para Sebastián SOLER, se podrá aplicar la atenuante de emoción violenta cuando el sujeto posea un estado psíquico que arrastra al sujeto y genera la disminución de los frenos inhibitorios (SOLER, 1956: 60).

Este concepto caracterizando la «violencia» de la emoción también ocupa un lugar central en las decisiones de los tribunales. Así, por ejemplo, la Cámara Nacional de Casación penal, causa núm. 1282/13 - Sala I, Bracamonte, Simón Jorge s/recurso de casación, refiriéndose a esta figura establece que

los requisitos específicos del tipo penal son el estado emocional — conmoción del ánimo que genera una modificación en la personalidad—, la violencia en la emoción —dificultad de controlar los impulsos y disminución marcada de los frenos inhibitorios.

En un sentido similar, el Tribunal Supremo español (Sentencia: núm. 417/2009 de fecha 07/04/2009) establece que una de las condiciones para aplicar la atenuante de arrebato u obcecación es: 
En efecto esta circunstancia de atenuación concurre cuando la obcecación y el arrebato al actuar entrañan una reducción de la imputabilidad provocada por situaciones que disminuyen la razonabilidad del pensamiento o el control de la voluntad debida a un duradero oscurecimiento u ofuscación del ánimo (S. 1696/2002, de 14 de octubre). Sus elementos son: a) Desde el punto de vista interno una situación de cólera o ímpetu pasional que reduzca limitando las facultades mentales del sujeto activo del delito, de modo que se produzca una situación de ofuscación de una importante entidad que suponga que sus resortes inhibitorios se vean seriamente afectados (cursivas añadidas).

Un rasgo que vale la pena resaltar es que aunque no siempre que experimentamos una emoción la manifestamos, en ocasiones, por ejemplo ante ira violenta, un hombre no puede poseer esa emoción y no manifestarse. Para decirlo de modo más simple, no puedo estar tranquilo si estoy bajo la «fuerza» de una ira que me nubla el entendimiento. Cada vez que esté bajo los efectos de esa emoción reaccionaré (KenNy, 2003: 43).

Por supuesto, aquí no quiero afirmar que este criterio sea el único elemento necesario para disminuir la responsabilidad tanto en casos de emoción violenta como en el arrebato u obcecación. Solo pretendo resaltar la importancia que posee la intensidad como impulso en nuestra atribución de responsabilidad.

En segundo lugar, el criterio de intensidad como motivo $b$ ), una emoción será más fuerte que otra si posee una mayor influencia en los planes de vida del agente, i. e. en la acción voluntaria. En palabras de KENNY, «bajo este criterio, cuán poderosa es una emoción depende de cuánto del comportamiento de un hombre pueda ser explicado mediante la referencia a ello» (KENNY, 2003: 24).

En principio, cuando la emoción es una reacción a cierto estímulo externo (por ejemplo, una serpiente) es mejor utilizar el primer criterio y, cuando el objeto de la emoción está a distanciado en el tiempo y el espacio es más útil tomar en consideración el segundo criterio. Por ejemplo, una persona que posee una gran ambición se comportará de determinada manera ante ciertas situaciones durante toda su vida. Este criterio de intensidad también entra en consideración al momento de atribuir responsabilidad penal. Así, podemos afirmar que un agente actuó por odio racial si se comporta de determinada manera ante personas de diferente raza a la de él.

Ambos criterios son independientes y, por tanto, en ocasiones, pueden entrar en conflicto. Sin embargo ellos pueden utilizarse para analizar la misma emoción. Por ejemplo, el miedo (a la oscuridad o a las alturas) puede medirse bajo ambos criterios. Si entro a lugares oscuros la intensidad de mi emoción se medirá por el movimiento de mi cuerpo, el aumento de palpitaciones, el temblor de piernas, etc. Si mido la intensidad de mi temor por el impacto en mi comportamiento veremos que nunca me expuse a lugares oscuros, $\mathrm{o}$ nunca fui a escalar montañas o esquiar si le tengo miedo a las alturas (KENNY, 2003: 25).

Este criterio de intensidad de la emoción es utilizado en la práctica judicial. Por ejemplo, el juez de instrucción (Zelaya) imputó por delito de homicidio calificado por odio al género a Lucas Adrián Azcona. Azcona mató a puñaladas a una estudiante a la salida del metro. Entre las razones para aplicar el agravante dijo «el odio es un sentimiento que el homicida viene alimentando en el tiempo con proyección general».

También consideró como síntoma de su misoginia la figura que tiene tatuada en su cuerpo y que había atacado con anterioridad, y posterioridad al caso a otras mujeres con arma blanca. Es decir, habría realizado conductas y comportamientos que se 
proyectan a lo largo del tiempo y muestran la importancia e intensidad que posee está emoción, entendida como motivo, para el sujeto.

Al igual que afirmé en el criterio anterior, no quiero decir que por esa razón el homicidio por motivos abyectos está justificado ${ }^{17}$. Solo pretendo señalar que en la práctica de atribución de responsabilidad penal se utilizan ambos criterios para medir la intensidad de la emoción. Y que esa práctica puede explicarse de modo coherente con la estructura de la acción humana en general y las emociones en particular.

Alguien podría afirmar que hablar de diferentes criterios para medir la intensidad de la emoción es un modo elíptico de afirmar que hay dos conceptos de emoción en juego. Sin embargo, ello no esa sí. Pensemos por ejemplo, dos criterios para medir el tamaño de una persona. El primer criterio es el peso y el segundo la edad. Estos criterios son independientes uno de otros y en ocasiones pueden oponerse. Así, un niño obeso puede, a pesar de su corta edad, tener un gran tamaño en el primer sentido pero no en el segundo. Otra persona puede tener muchos años y pesar $40 \mathrm{~kg}$. Así bajo un criterio diremos que es pequeño y otro diremos que es grande. Ello no quiere decir que estemos utilizando diferentes conceptos de lo que es una persona.

En síntesis, en este apartado mostré un modo adecuado de entender por qué en ocasiones las emociones se utilizan para atenuar y en otras para agravar la pena en muchos códigos penales. Esta manera de entender los diferentes sentidos de intensidad de una emoción explica de modo racional nuestras prácticas de atribución de responsabilidad.

\section{CONCLUSIONES}

A lo largo de este trabajo intenté presentar un panorama conceptual de la discusión sobre las emociones. Mi principal objetivo al hacerlo fue resaltar que esta disputa entre teorías mecanicistas y teorías cognitivas o cognitivas evaluativas está planteada como exageraciones de ciertos rasgos que poseen las emociones. El objetivo central fue mostrar que estas teorías no son rivales, como tradicionalmente se las ha considerado, sino que más bien cada una se enfoca en diferentes aspectos de las emociones. Por esa razón, puede afirmarse que sostuve una teoría dual de las emociones.

La estrategia para argumentar a favor de la concepción dual de las emociones fue utilizar el esquema esgrimido por VON WRIGHT para la relación entre mente y materia. Presenté los diferentes tipos de prioridades para diferentes aspectos de las emociones: i) hay una prioridad epistémica de lo mental sobre lo neuronal; ii) hay una prioridad causal de lo neuronal sobre lo conductual, y iii) existe una prioridad semántica de lo conductual sobre lo mental.

En la última parte de este trabajo sostuve que hay dos criterios para medir la intensidad de la emoción: i) intensidad como impulso, y ii) intensidad como motivo. Estos diferentes criterios se manifiestan en los códigos penales, los primeros en general como atenuantes de la responsabilidad y los segundos como agravantes. Por supuesto, no

17 Aunque creo que la agravante está justificada no pretendo analizar las razones de por qué ello es así. Para un estudio de estos temas vid., por ejemplo, PERALTA, 2012. 
intenté justificar estás figuras sino más bien mostrar que ellas pueden ser reconstruidas de modo racional y no solo como un mero deseo de castigo o utilización de diferentes teorías para diferentes tipos de casos según nuestras intuiciones o reacciones frentes a ciertas clases de conductas.

\section{BIBLIOGRAFÍA}

CARRARA, F, 1957: Programa de derecho criminal, Temis.

DAMASIO, A., 1994: Descartes' error-Emotion, Reason and the Human Brain, New York: Avon Books.

Davidson, D., 1980: Ensayos sobre acciones y sucesos, México: Instituto de Investigaciones Filosóficas.

DwORKIN, R., 1977: Los derechos en serio, España: Planeta.

Elster, J, 1998: Alquemies of the Mind-Rationality and the Emotions, Cambridge: Cambridge University Press.

GonZÁleZ LAGIER, D., 2009: Emociones, responsabilidad y derecho, Madrid: Marcial Pons.

Hart, H. L. A., 1963: El concepto de Derecho, Buenos Aires: Abeledo Perrot.

Hume, D, 1739: Tratado de la naturaleza bumana, Libro II, Parte Primera, Sección V.

Kahan, D., y Nussbaum, M., 1996: «Two Conceptions of Emotions in Criminal Law», Columbia Law Review, 96 (2): 269-374.

Kenny, A., 2003: Action, Emotion and Will: London y New York: Routledge.

LYONS, W., 1993: Emoción, Barcelona: Anthropos.

Nussbaum, M., 2006: El ocultamiento de lo bumano. Repugnancia, vergüenza y ley, Buenos Aires: Katz.

Peralta, J., 2012: Motivos reprochables, Barcelona: Marcial Pons.

Platón: «Fedro», en P. DE AzCÁRATE (ed.), Platón. Obras Completas, 1871: II, Madrid: Medina y Navarro.

RyLE, G., 1949: The Concept of Mind, London: Hutchinson.

— 2009: «Feelings», Collected Papers (II), New York: Routledge, 284-299.

SCHIMILL, U., 2010: Las implicaturas del resentimiento — La tragedia de Otelo-, México: Themis.

SOLER, S., 1956: Derecho penal argentino, Buenos Aires: Tipográfica editora argentina.

Solomon, R., 2003: What is an Emotion, Oxford: Oxford University Press.

SutHeRland, S., 1976: «Hume on Morality and Emotions», The Philosophical Quaterly, 26: 14-23.

TAdross, V., 2004: «Attribution, Ethics and Emotions», en Criminal Responsibility. Review Article' in Modern Law Review, 67, 332.

Von Wright, G. H., 1970: Norma y acción, Madrid: Tecnos.

- 1980: Explicación y comprensión, Madrid: Alianza editorial.

- 1998a: «On Mind and Matter», In the Shadow of Descartes: Essays in the Philosophy of Mind, Netherlands Kluwer Academia.

- 1998b: «Sensations and Perceptions», In the Shadow of Descartes: Essays in the Philosophy of Mind, Netherlands Kluwer Academia.

— 2003: «Valor, norma y acción», Doxa, 26: 53-79.

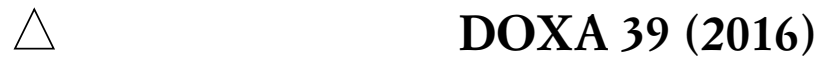

